\title{
USO DE SEMENTES DE ACEROLA COMO BIOSSORVENTE PARA REMOÇÃO DE Cr(VI) DE SOLUÇÕES AQUOSAS
}

\author{
J. C. T. de REZENDE ${ }^{1}$, V. H. dos S. RAMOS ${ }^{1}$, D. D. A. NUNES ${ }^{1}$, E. N. dos REIS ${ }^{1}$, E. de JESUS ${ }^{1}$, J. \\ A. PACÍFICO ${ }^{1}$ e D. C. SILVA ${ }^{1}$ \\ ${ }^{1}$ Universidade Federal de Sergipe, Departamento de Engenharia Química \\ E-mail para contato: jessik_cristian@hotmail.com
}

\begin{abstract}
RESUMO - Este trabalho teve como objetivo avaliar o potencial de utilização de sementes de acerola (Malpighia emarginata) na adsorção de $\mathrm{Cr}(\mathrm{VI})$ de soluções aquosas. $\mathrm{Na}$ obtenção do biossorvente foi utilizado tratamento térmico à temperatura de $170{ }^{\circ} \mathrm{C} \mathrm{e}$, em seguida, o biossorvente foi triturado. A determinação de $\mathrm{Cr}(\mathrm{VI})$ foi feita por espectrofotometria UV-visível através da complexação com 1,5-difenilcarbazida, medindo a absorbância no comprimento de onda de $540 \mathrm{~nm}$. Foram realizados experimentos variando o tempo de contato e o pH inicial. A eficiência da remoção de íons de $\mathrm{Cr}(\mathrm{VI})$ foi avaliada por espectroscopia de infravermelho. A remoção média do biossorvente utilizado foi de $66 \%$.
\end{abstract}

\section{INTRODUÇÃO}

A acerola (Malpighia emarginata) tem grande importância nutricional por ser fonte natural de vitamina C. Aliado ao aspecto nutricional e funcional do fruto, a acerola apresenta uma elevada produção e é bastante utilizada na região Nordeste do Brasil na fabricação de polpa de fruta, sorvete e suco, apresentando forte potencial para industrialização. Ressalta-se que as cascas e as sementes possuem pouco aproveitamento, contribuindo desta forma para a geração de resíduos sólidos (MELO et al., 2009).

Os processos convencionais para remoção de metais pesados apresentam custos elevados o que vem favorecendo o aparecimento de novas tecnologias e métodos de redução/eliminação dos poluentes. Uma dessas alternativas é a biossorção que consiste na remoção de poluentes dos efluentes utilizando materiais de origem biológica, que após podem ser utilizados, por exemplo, em fornos de cimentos como forma de tratamento do resíduo. Este processo pode torna-se vantajoso, uma vez que combina boa eficiência de remoção dos poluentes com custos de implantação e operação reduzidos (PINA, 2011).

O cromo hexavalente, $\mathrm{Cr}(\mathrm{VI})$, é tóxico e carcinogênico por causa do elevado potencial de oxidação e por sua elevada capacidade de penetrar em membranas biológicas. Excessiva exposição ao $\mathrm{Cr}(\mathrm{VI})$ pode causar várias doenças, incluindo danos ao fígado, rins, sistema circulatório, tecidos nervosos e sistema sanguíneo (MELO, 2008). Atualmente, existem fortes restrições governamentais relativamente à deposição de águas residuais. O Conselho Nacional do Meio Ambiente (CONAMA) 
através da Resolução N²0, de 18 de julho de 1986, estabeleceu os valores máximos permissíveis de alguns metais em que para $\mathrm{Cr}(\mathrm{III})$ e $\mathrm{Cr}(\mathrm{VI})$ são, respectivamente, $1,0 \mathrm{mg} \mathrm{L}^{-1}$ e $0,5 \mathrm{mg} \mathrm{L}^{-1}$.

Diante do exposto, este trabalho buscou avaliar a potencialidade do uso de sementes de acerola na remoção de $\mathrm{Cr}(\mathrm{VI})$. O estudo foi realizado em batelada visando identificar o percentual de remoção e o comportamento cinético da adsorção de $\mathrm{Cr}(\mathrm{VI})$ pelo biossorvente.

\section{MATERIAIS E MÉTODOS}

\subsection{Preparação do biossorvente}

As sementes de polpas de frutas foram cedidas pela indústria Pomar do Brasil Indústria e Comércio de Alimentos Ltda, localizada no bairro Distrito Industrial, em Aracaju-SE. As sementes foram coletadas em sacos plásticos e levadas ao Laboratório de Química Industrial (LQI) da Universidade Federal de Sergipe, onde foram devidamente lavadas com água para eliminação de restos de polpa. As sementes foram secas em mufla (GP Científica) a $170{ }^{\circ} \mathrm{C}$, sendo em seguida trituradas em um processador de alimentos. A granulometria do biossorvente foi feita utilizando peneiras de 4, 9, 12, 32, 100 e 200 mesh e classificador granulométrico da Bertel.

\subsection{Determinação de acidez}

1,0 g do pó do biossorvente 12 mesh foi misturado em $50 \mathrm{~mL}$ de água destilada. A mistura foi agitada utilizando agitador magnético durante 24 horas. Logo após, a mistura foi filtrada, sendo 10 $\mathrm{mL}$ do filtrado titulado com $\mathrm{NaOH} 0,1 \mathrm{~mol} \mathrm{~L}^{-1}$, utilizando fenolftaleína como indicador segundo procedimento do Instituto Adolfo Lutz (2008).

\subsection{Determinação de $\operatorname{Cr}(V I)$}

A solução concentrada de $\mathrm{Cr}(\mathrm{VI})$ foi preparada a partir de dicromato de potássio (Merk) dissolvendo 2,829 $\mathrm{g} \mathrm{em} 1000 \mathrm{~mL}$ de água destilada, formando assim, uma solução de $1000 \mathrm{mg} \mathrm{L}^{-1} \mathrm{de}$ $\mathrm{Cr}(\mathrm{VI})$. Foram obtidas, por diluições, soluções nas concentrações padrões de 0,$2 ; 0,4 ; 0,6 ; 0,8$ e 1,0 $\mathrm{mg} \mathrm{L}^{-1}$ para determinação da curva de calibração, segundo procedimento de determinação de $\mathrm{Cr}(\mathrm{VI})$ por complexação 1,5-difenilcarbazida em meio ácido (Morita e Assumpção, 2007). A absorbância foi medida no espectrofotômetro (UV-VIS da VARIAN) no comprimento de onda igual a $540 \mathrm{~nm}$. A concentração de $\mathrm{Cr}$ (VI) após a adsorção foi determinada usando o valor da absorbância da amostra. A porcentagem de cromo hexavalente removida foi determinada pela Equação 1.

\% Remoção de $\operatorname{Cr}(\mathrm{VI})=\left[\frac{\left(\mathrm{C}_{\mathrm{i}}-\mathrm{C}_{\mathrm{f}}\right)}{\mathrm{C}_{\mathrm{i}}}\right] \times 100$ 
O estudo do efeito do tempo de contato na remoção de $\mathrm{Cr}(\mathrm{VI})$ foi realizado na concentração inicial de $\mathrm{Cr}(\mathrm{VI}) 5 \mathrm{mg} \mathrm{L}^{-1}$, quantidade de biossorvente $0,1 \mathrm{~g}(12 \mathrm{mesh})$; temperatura $25 \pm 1^{\circ} \mathrm{C}$ e pH 2. A concentração de $\mathrm{Cr}(\mathrm{VI})$ adsorvido foi medida na faixa de tempo de 10 a $140 \mathrm{~min}$.

$\mathrm{O}$ estudo da influência do $\mathrm{pH}$ na remoção do $\mathrm{Cr}(\mathrm{VI})$ foi realizada com ajuste do $\mathrm{pH}$ inicial da solução em 2, 5, 7 e 8, concentração de $\mathrm{Cr}(\mathrm{VI}) \mathrm{de} 5 \mathrm{mg} \mathrm{L}^{-1}$, massa do biossorvente $0,1 \mathrm{~g}$ (12 mesh) e tempo de equilíbrio de $120 \mathrm{~min}$.

\subsection{Cinética de adsorção}

Vários modelos cinéticos são utilizados para examinar o mecanismo controlador do processo de adsorção, tais como, reação química, controle da difusão e transferência de massa. Contudo, os modelos empregados com maior frequência são o pseudoprimeira ordem e o pseudosegunda ordem. $\mathrm{O}$ modelo pseudoprimeira ordem é representado pela Equação 2:

$$
\frac{\mathrm{dQ}}{\mathrm{dt}}=\mathrm{k}_{1}\left(\mathrm{Q}_{\mathrm{e}}-Q_{t}\right)
$$

em que $\mathrm{k}_{1}$ é a constante de adsorção de pseudoprimeira ordem $\left(\min ^{-1}\right), \mathrm{Q}_{\mathrm{e}}$ e $\mathrm{Q}_{\mathrm{t}}$ são as quantidades adsorvidas por grama de biossorvente no equilíbrio e no tempo $\mathrm{t}$, respectivamente $\left(\mathrm{mmols}^{-1}\right)$. Integrando a Equação 1 de $\mathrm{Q}_{\mathrm{t}}=0$ em $\mathrm{t}=0$ a $\mathrm{Q}_{\mathrm{t}}=\mathrm{Qe}$ em $\mathrm{t}=\mathrm{t}$ obtém-se a Equação 3:

$$
\ln \left(\mathrm{Q}_{\mathrm{e}}-\mathrm{Q}_{\mathrm{t}}\right)=\ln \mathrm{Q}_{\mathrm{e}}-\mathrm{k}_{1} \mathrm{t}
$$

$\mathrm{O}$ valor de $\mathrm{k}_{1}$ e de $\mathrm{Q}_{\mathrm{e}}$ não experimental podem ser obtidos do gráfico $\ln \left(\mathrm{Q}_{\mathrm{e}}-\mathrm{Q}_{\mathrm{t}}\right)$ versus $\mathrm{t}$.

O modelo pseudosegunda ordem é representado na Equação 4:

$$
\frac{\mathrm{dQ}_{\mathrm{t}}}{\mathrm{dt}}=\mathrm{k}_{2}\left(\mathrm{Q}_{\mathrm{e}}-Q_{t}\right)^{2}
$$

em que $\mathrm{k}_{2}$ é a constante da taxa de adsorção de pseudosegunda ordem $\left(\mathrm{g} \mathrm{mg}^{-1} \mathrm{~min}^{-1}\right)$.

Integrando a Equação 4 nos moldes da Equação 2 e linearizado-a tem-se a Equação 5: 


$$
\frac{\mathrm{t}}{Q_{t}}=\frac{1}{k_{2} Q_{e}^{2}}+\frac{1}{\mathrm{Q}_{\mathrm{e}}}
$$

De acordo com Weber e Moris se a difusão intrapartícula for fator determinante da velocidade de adsorção, os dados cinéticos experimentais devem seguir a Equação 6:

$$
\mathrm{Q}_{\mathrm{t}}=\mathrm{k}_{\mathrm{dif}} \mathrm{t}^{\frac{1}{2}}+C
$$

sendo $\mathrm{Q}_{\mathrm{t}}$ a quantidade de $\mathrm{Cr}(\mathrm{VI})$ adsorvidade (mmols $\mathrm{g}^{-1}$ ), $\mathrm{t}$ o tempo de adsorção (min), C (mmols $\mathrm{g}^{-1}$ ) uma constante relacionada coma resistência à difusão intrapartícula e $\mathrm{k}_{\mathrm{dif}}$ é o coeficiente de difusão intrapartícula (mmols $\mathrm{g}^{-1} \mathrm{~min}^{-1 / 2}$ ). Este modelo fornece informações sobre os mecanismos de transferência de massa envolvidos no processo de adsorção, por exemplo, se ocorre difusão intrapartícula, se há ou não formação de camada limite externa.

\subsection{Espectroscopia de absorção na região do infravermelho (FTIR)}

As amostras foram analisadas na forma de pastilhas de $\mathrm{KBr}$ utilizando o equipamento Nicolet, modelo i-10 FTIR, com acessório SMART-OMNI, na região compreendida entre 400 e $4000 \mathrm{~cm}^{-1}$ com resolução de $4 \mathrm{~cm}^{-1}$.

\section{RESULTADOS E DISCUSSÃO}

A acidez média de $12 \%$ obtida para as sementes de acerola é decorrente de ácidos carboxílicos. A Figura 1 do espectro FTIR do pó de sementes de acerola indicam a presença destes ácidos no biomaterial. As análises FTIR foram realizadas no biossorvente antes e depois da adsorção. Nota-se pelo espectro que provavelmente todos os grupos da superfície do biomaterial estiveram envolvidos na adsorção, havendo aumento na transmitância no comprimento de onda dos grupos identificados, talvez em decorrência da interação com a espécie metálica. As bandas largas e fortes em 3450,2 e $3416,1 \mathrm{~cm}^{-1}$ foram atribuídas ao grupo hidroxila $(-\mathrm{OH})$, provavelmente de ácidos carboxílicos, o que pode indicar que este último grupo foi fortemente envolvido na biossorção (BARBOSA, 2008). 


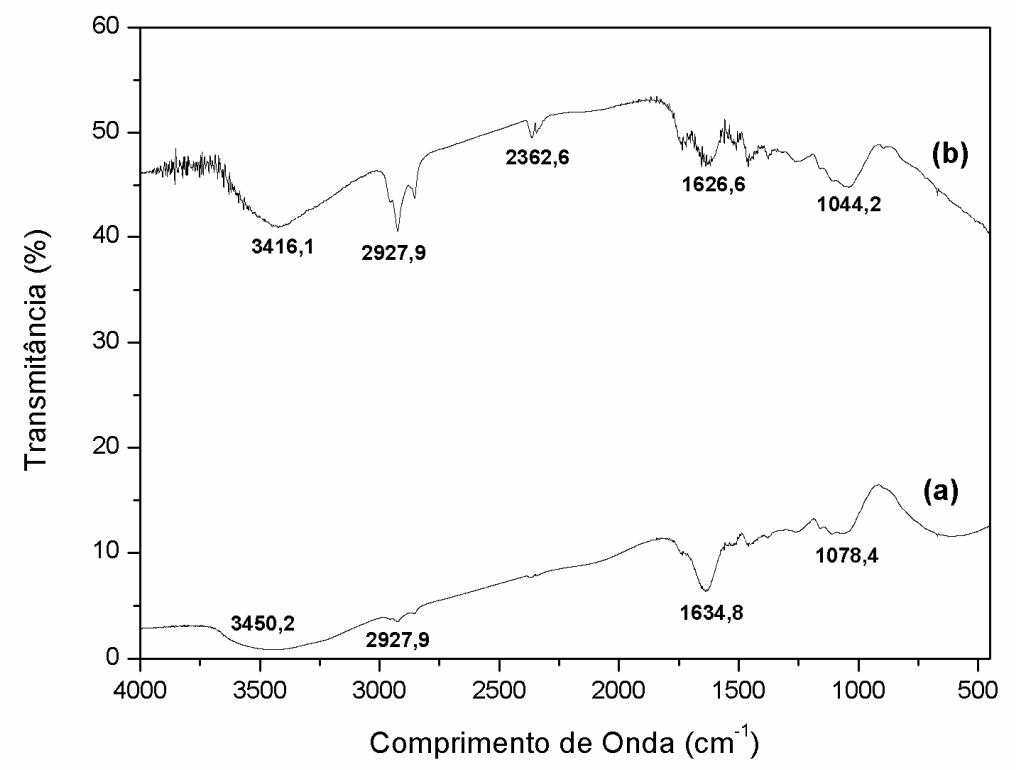

Figura 1: Espectro FTIR para o biossorvente (pó de acerola), sendo (a) antes da adsorção e (b) após à adsorção.

O pico em 2927,9 $\mathrm{cm}^{-1}$ indica o estiramento de ligações $\mathrm{C}-\mathrm{H}$ de alcanos ou grupo alquila (BARBOSA, 2008). As bandas de 1634,8 e 1626,6 $\mathrm{cm}^{-1}$ foram atribuídas à vibração da carbonila $(\mathrm{C}=\mathrm{O})$ de ácidos carboxílicos (BARBOSA, 2008). Os picos em 1078,4 e 1044,2 $\mathrm{cm}^{-1}$ são referentes ao estiramento de ligações C-O, provavelmente de ácidos carboxílicos (SILVERSTEIN et al., 2005). Nota-se que, após à adsorção, surgiu um novo pico de $2362,6 \mathrm{~cm}^{-1}$ que pode indicar a presença de interações Cr-CO (SHUKLA, 2012).

A Figura 2 mostra que o tempo de equilíbrio foi de 120 minutos, sendo este o tempo de contato utilizado para se determinar qual o melhor $\mathrm{pH}$ inicial para a remoção de $\mathrm{Cr}(\mathrm{VI})$. $\mathrm{O}$ equilíbrio na remoção de $\mathrm{Cr}(\mathrm{VI})$ pode ser explicado pela utilização de todo os sítios ativos do biomaterial. Durante a adsorção, o cromo hexavalente se impregna na superfície do adsorvente até que toda a superfície esteja coberta de íons do metal. Neste ponto a concentração de $\mathrm{Cr}$ (VI) deixa de diminuir e se mantém em equilíbrio com o adsorvente em solução. Com isso, o tempo de equilíbrio da adsorção varia de acordo com a superfície do adsorvente utilizado (RAMOS et al., 2013). 


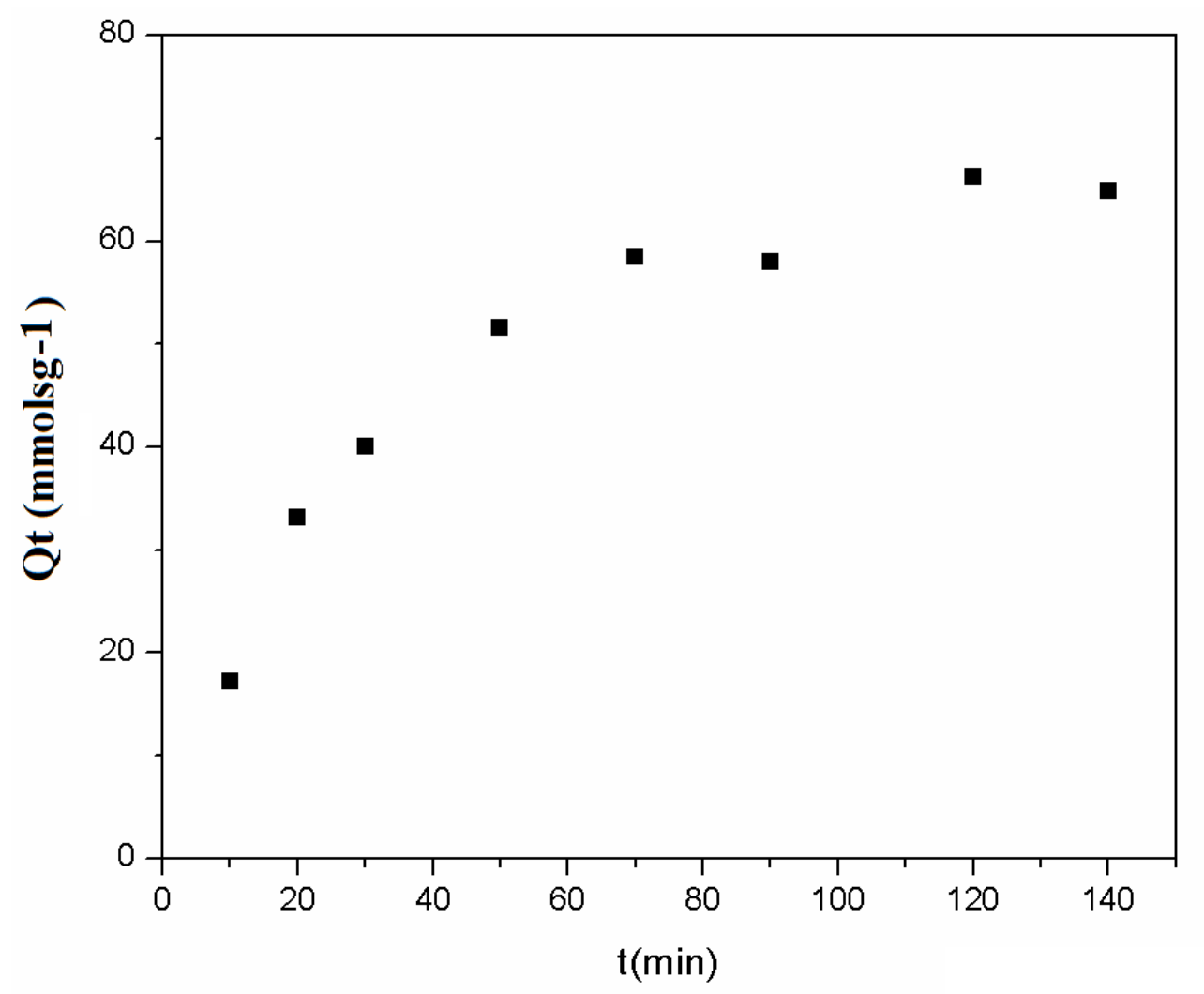

Figura 2: Efeito do tempo de contato na remoção de $\mathrm{Cr}(\mathrm{VI})$. Condições: concentração inicial de $\mathrm{Cr}(\mathrm{VI}) 5 \mathrm{mg} \mathrm{L}^{-1}$; volume do meio, $100 \mathrm{~mL}$; quantidade de adsorvente, $0,1 \mathrm{~g}$; temperatura $25 \pm 1{ }^{\circ} \mathrm{C}$; velocidade de agitação de $150 \mathrm{rpm} ; \mathrm{pH} 2$ para o pó da semente de acerola.

$\mathrm{O}$ pH é um dos parâmetros que interferem no potencial de adsorção. $\mathrm{O}$ pH afeta não só a carga superficial de biossorvente, mas também o grau de ionização durante a reação. No pH inicial 2,0 houve remoção de $66 \%$ de $\mathrm{Cr}(\mathrm{VI})$ da solução aquosa, enquanto no $\mathrm{pH}$ inicial 5,7 e 8 não houve remoção. A literatura mostra resultados semelhantes na remoção de metais em $\mathrm{pH}$ ácido, a exemplo de folhas de Araucária secas (SHUKLA, 2012), casca de nozes (ALTUN e PEHLIVAN, 2012), carvão ativado granular (SOUZA et al., 2009), casca de coco (PINO, 2005), algas marinhas (PINA, 2012), raízes de jacinto e folhas de nim (SINGHA e DAS, 2010), pó de quiabo (CONCEIÇÃO et al., 2014), casca e polpa de laranja Osage (PEHLIVAN, 2012).

No estudo cinético, verificou-se que o melhor ajuste foi utilizando o modelo pseudosegunda ordem (Figura 3), em que se obteve $\mathrm{R}^{2}=0,9823$, valor de $\mathrm{k}_{2}$ de $0,3958 \mathrm{~g} \mathrm{mmols}{ }^{-1} \min ^{-1}$ e $\mathrm{Q}_{\mathrm{e}}$ calculado de $0,0787 \mathrm{mmols} \mathrm{g}^{-1}$. Utilizando o modelo de adsorção de Weber e Moris obteve-se $\mathrm{R}^{2}$ igual a 0,9200 , $\mathrm{k}_{\text {dif }}$ de $0,0063 \mathrm{mmols} \mathrm{g}^{-1} \mathrm{~min}^{-1 / 2}$ e C igual a $0,0018 \mathrm{mmols} \mathrm{g}^{-1}$. Como o valor de $\mathrm{C}$ foi diferente de zero e os dados experimentais pouco se ajustaram ao modelo intrapartícula de Weber e Morris, sugere-se que o mecanismo de transferência de massa durante a adsorção do $\mathrm{Cr}(\mathrm{VI})$ provavelmente é 
dominado pela formação de uma camada limite externa (Rocha et al., 2012), não excluindo contudo outros mecanismos.

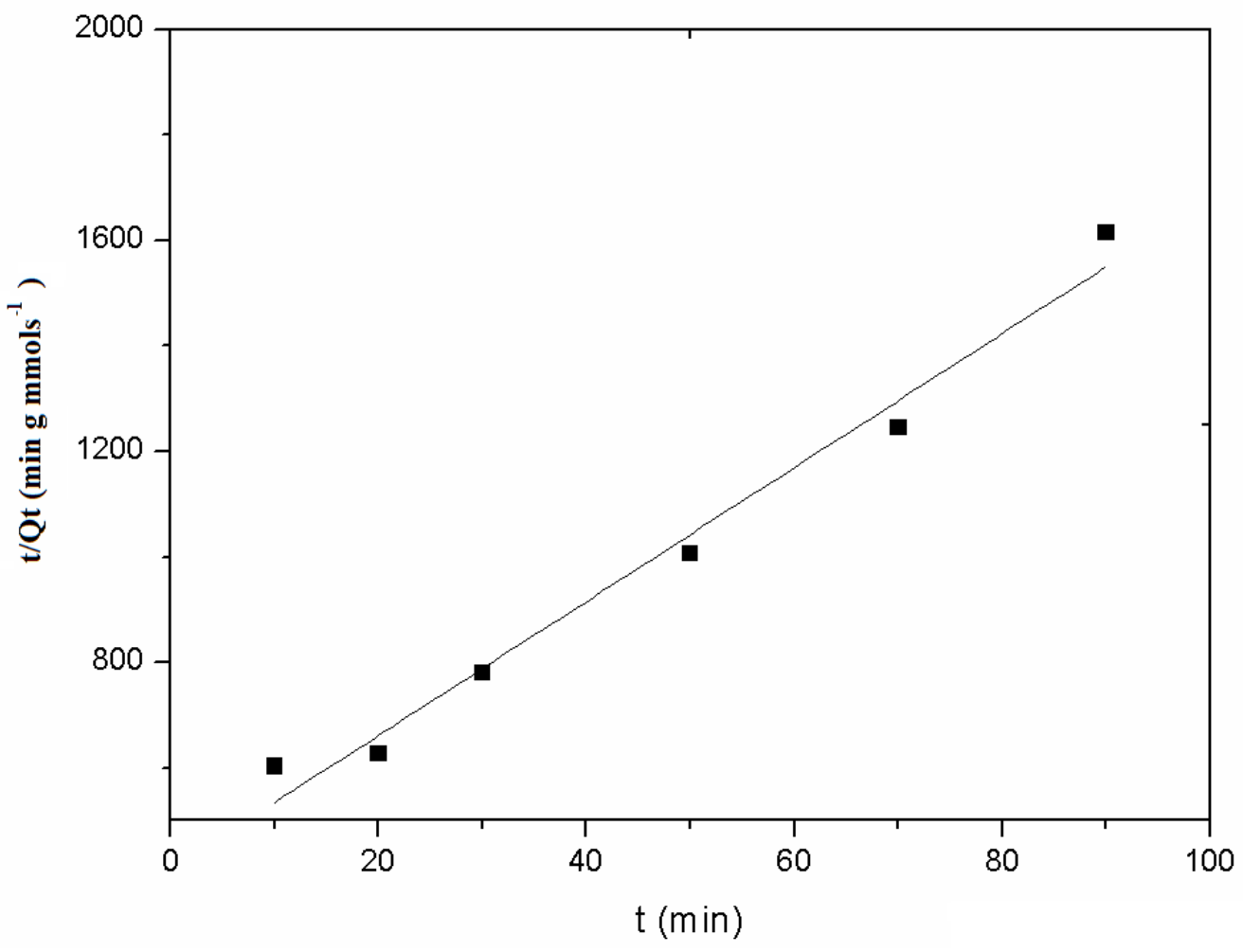

Figura 3: Cinética de adsorção pseudosegunda ordem (Equação 5).

\section{CONCLUSÃO}

Considerando os resultados apresentados, pode ser concluído que a remoção de Cr(VI) aumenta com o aumento do tempo de contato entre adsorvente e adsorvato até atingir um tempo de equilíbrio, sendo este 120 minutos para as sementes de acerola. A maior remoção de $\mathrm{Cr}(\mathrm{VI})$ foi de $66 \%$ utilizando $0,1 \mathrm{~g}$ de biossorvente (12 mesh) com $\mathrm{pH}$ inicial igual a 2. Verificou-se uma cinética rápida, sendo que em 120 min foi atingido equilíbrio. A transferência de massa durante a adsorção foi dominada pela formação de camada limite externa.

Por se tratar de material de baixo custo, considerado como resíduo da agroindústria, as sementes de acerola apresentam-se como uma alternativa viável no tratamento de contaminação por $\mathrm{Cr}(\mathrm{VI})$. 


\section{9 a 22 de outubro de 2014 \\ Florianópolis/SC}

\section{REFERÊNCIAS}

ALTUN, T.; PEHLIVAN, E.: Removal of Cr(VI) from aqueous solutions by modified walnuts shells. Food Chemistry, Vol.132(2), pp.693-700, 2012.

BARBOSA, L. C. A.; Espectroscopia no infravermelho na caracterização de compostos orgânicos. Editora UFV, $1^{\circ}$ reimpressão. Viçosa, MG: UFV, 2008.

CONAMA, Resolução $\mathrm{N}^{\circ}$ 20, de 18 de julho de 1986, in: MOTA, S.: Preservação e Conservação de Recursos Hídricos. Rio de Janeiro: ABES, 1995.

CONCEIÇÃA, J. C.; RAMOS, V. H. S.; JESUS, E.; SILVA, A. S.; COSTA, A. W. M. C.: Biosortion of $\mathrm{Cr}(\mathrm{VI})$ from Aqueous Solutions Using Chemically Modified Okra Powder. Journal of Basic \& Applied Sciences, 10, 73-79, 2014.

INSTITUTO ADOLFO LUTZ. Normas analíticas do Instituto Adolfo Lutz. v. 1: Métodos químicos e físicos para análise de alimentos, IV edição, $1^{\text {a }}$ Edição Digital. São Paulo, 2008. P. 25-26.

MELO, K. R. O.; Estudo da digestão de sedimento de curtume visando a extração de cromo por microemulsão. Tese (Doutor em Engenharia Química). Natal, RN: UFNR, 2008.

MELO, E. A.; CAETANO, A. C. S.; LIMA, V. L. A. G.; MACIEL, M. I. S.; ARAÚJO, C. R.: Extração de antioxidantes de resíduos agroindustriais de acerola. Brazilian Journal Food Technology, v. 12, n. 2, p. 155-160, abr./jun. 2009.

MORITA, T.; ASSUMPÇÃO, R. M. V.; Manual de soluções, reagentes e solventes: padronização, preparação, purificação com indicadores de segurança e de descarte de produtos químicos. São Paulo: Editora Blucher, 2007.

PEHLIVAN, E.; PEHLIVAN, E.; TUTAR, H.K.; Hexavalent chromium removal by Osage Orange. Food Chemistry, Vol.133(4), p. 1478-1484, 2012.

PINA, F. D. S.; Tratamento de águas contaminadas com crómio(VI) por bioadsorção em algas marinhas. 2011. 65f. Tese (Mestre em Engenharia do Ambiente), Faculdade de Engenharia da Universidade do Porto, Porto, Portugal, 2011.

PINO, G. A. H.; Biossorção de metais pesados utilizando pó da casca de coco cerde (Cocos nucifera). Tese (Mestre em Engenharia Metalúrgica). Rio de Janeiro, RJ: PUC-Rio, 2005.

RAMOS, V. H. S.; CONCEIÇÃO, J. C.; JESUS, E.; MARQUES, J. J.; SILVA, A. S.; SILVA, D. C.: Remoção de cromo hexavalente por biossorção usando pó de quiabo. XXXVI Congresso Brasileiro de Sistemas Particulados. Universidade Federal de Alagoas. Maceió-AL, 2013.

ROCHA, O. R. S.; NASCIMENTO, G. E.; CAMPOS, N. F.; SILVA, V. L.; DUARTE, M. M. M. B.: Avaliação do processo adsortivo utilizando mesocarpo de coco verde para remoção do corante cinza reativo BF-2R. Quim. Nova, Vol. 35, No. 7, 1369-1374, 2012.

SHUKLA, D.; VANKAR, P.; Efficient biossorption of chromium(VI) ion by dry Araucaria leaves. Environmental Science and Pollution Research, Vol.19(6), pp.2321-2328, 2012.

SILVERSTEIN, R. M.; WEBSTER, F. X.; KIEMLE, D. J.: Spectrometric identification of organic compouds. John Wilwy \& Sons, Seventh Edition. State University of New York, 2005.

SINGHA, M., DAS, S.; Cr(VI) adsorption - mechanistic approach for biosorption from aqueous solutions. The Bioscan: Special issue, Vol. 2; 509-514. Índia, 2010.

SOUZA, R. S.; CARVALHO, S. M. L.; GARCIA JÚNIOR, M. L. R.; SENA, R. S. F.; Adsorção de cromo (VI) por carvão ativado granular de soluções diluídas utilizando um sistema batelada sob pH controlado. ACTA Amazonica, vol. 39(3), 661 - 668, 2009. 